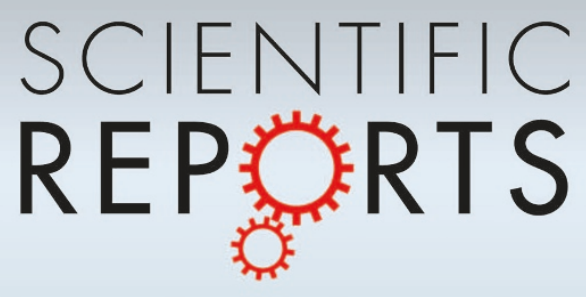

OPEN

SUBJECT AREAS:

PHASE TRANSITIONS

AND CRITICAL

PHENOMENA

MATERIALS SCIENCE

MAGNETIC PROPERTIES AND

MATERIALS

FERROELECTRICS AND

MULTIFERROICS

Received

3 July 2013

Accepted

29 July 2014

Published

19 September 2014

Correspondence and requests for materials should be addressed to

T.K. (kambe@science. okayama-u.ac.jp)

\section{Dynamically fluctuating electric dipole moments in fullerene-based magnets}

\author{
Takashi Kambe \& Kokichi Oshima \\ Department of Physics, Faculty of Science, Okayama University, Okayama 700-8530, Japan.
}

We report here the direct evidence of the existence of a permanent electric dipole moment in both crystal phases of a fullerene-based magnet-the ferromagnetic $\alpha$-phase and the antiferromagnetic $\alpha^{\prime}$-phase of tetra-kis-(dimethylamino)-ethylene- $\mathrm{C}_{60}\left(\mathrm{TDAE}-\mathrm{C}_{60}\right)$-as determined by dielectric measurements. We propose that the permanent electric dipole originates from the pairing of a TDAE molecule with surrounding $\mathrm{C}_{60}$ molecules. The two polymorphs exhibit clear differences in their dielectric responses at room temperature and during the freezing process with dynamically fluctuating electric dipole moments, although no difference in their room-temperature structures has been previously observed. This result implies that two polymorphs have different local environment around the molecules. In particular, the ferromagnetism of the $\alpha$-phase is founded on the homogeneous molecule displacement and orientational ordering. The formation of the different phases with respect to the different rotational states in the JahnTeller distorted $\mathrm{C}_{60} \mathrm{~s}$ is also discussed.

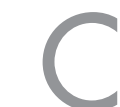

ontrol of the spatial arrangement of molecules plays a role in adjusting the magnetic interactions in molecule-based magnets ${ }^{1}$. The coherent magnetic interactions among the localized spins in a fullerene cage are essentially mediated by the cooperative molecular orbital (MO) ordering between the $\mathrm{C}_{60}$ cages via the Jahn-Teller (JT) distortion ${ }^{2}$. The threefold degeneracy of the lowest-unoccupied molecular orbital (LUMO) of the $\mathrm{C}_{60}$ cage is partly removed by the distortion of the $\mathrm{C}_{60}$ cage by intramolecular JT coupling ${ }^{3}$. The ferro(antiferro)-rotative orbital ordering of neighboring cages leads to the antiferro(ferro) magnetic correlation between the cages ${ }^{2,4-7}$. The orthogonally coupled wave functions of the nearest neighbors reduce the direct transfer of electrons, which is a favorable configuration for ferromagnetic interactions ${ }^{2}$. This type of close coupling between the spin and orbital degrees of freedom is a remarkable characteristic of fullerene-based magnets in comparison with other molecular magnets.

Tetra-kis-(dimethylamino)-ethylene- $\mathrm{C}_{60}$ (TDAE- $\mathrm{C}_{60}$ ) has two polymorphic crystals: the $\alpha$ and the $\alpha^{\prime}$ phases. The $\alpha$-phase is the first fullerene ferromagnet with a high transition temperature $\left(T_{\mathrm{C}}\right)$ of $16 \mathrm{~K}^{4,5,8}$. On the contrary, the $\alpha^{\prime}$-phase crystal undergoes antiferromagnetic ordering below the Neel temperature $\left(T_{\mathrm{N}}\right)$ of $7 \mathrm{~K}^{9}$. Our previous structural studies revealed that these different magnetic phase transitions for the two polymorphs are grounded in their structural peculiarities ${ }^{9-11}$. Only the $\alpha$-phase shows a structural phase transition at approximately $T_{\mathrm{S}}=170 \mathrm{~K}$. Below the $T_{\mathrm{S}}$, the low-temperature (LT) unit cell contains two crystallographically inequivalent $\mathrm{C}_{60}$ molecules in which the hexagons face each other. This configuration would provide alternatively coupled MOs along all of the nearest-neighbor directions, leading to the LT antiferro-rotative structure for JTdistorted $t_{1 \mathrm{u}}$ orbitals. Note that the orientational ordering in the $\alpha$-phase works in close cooperation with the cubical distortion of the surrounding TDAE molecules. By contrast, the $\alpha^{\prime}$-phase crystals show no evidence of a structural phase transition down to $25 \mathrm{~K}$. The unit cell of the $\alpha^{\prime}$-phase contains one $\mathrm{C}_{60}$ with hexagonalpentagonal contact between the nearest-neighbor $\mathrm{C}_{60}$ s. This configuration provides a ferro-rotative arrangement for the JT-distorted $t_{1 \mathrm{u}}$ orbitals within the $a b$-plane and along the $c$-axis. A schematic phase representation for the two polymorphs is shown in Fig. 1. Accordingly, the magnetism of TDAE- $\mathrm{C}_{60}$ closely correlates with the orientational freedom of the $\mathrm{C}_{60}$ cages.

In spite of the distinct differences in the LT structures and the LT magnetism, however, it has not been possible to distinguish the two polymorphs via room-temperature structural analysis ${ }^{9-11}$. As mentioned above, the LT structures for the two polymorphs have remarkable differences in their $\mathrm{C}_{60}$ orientations. Hence, this study determines the cause of the differences in the two polymorphs at high temperature by dielectric experiments. In this Letter, we present the dielectric measurement results for the two polymorphic crystals. The dielectric investigation uncovered clearly different dielectric responses in the high-temperature region above the orientational ordering temperature, indicating a distinct difference in the orientational dynamics of $\mathrm{C}_{60}$ for the two polymorphs. The observed dielectric behavior is responsible for a permanent electric dipole, which should be 


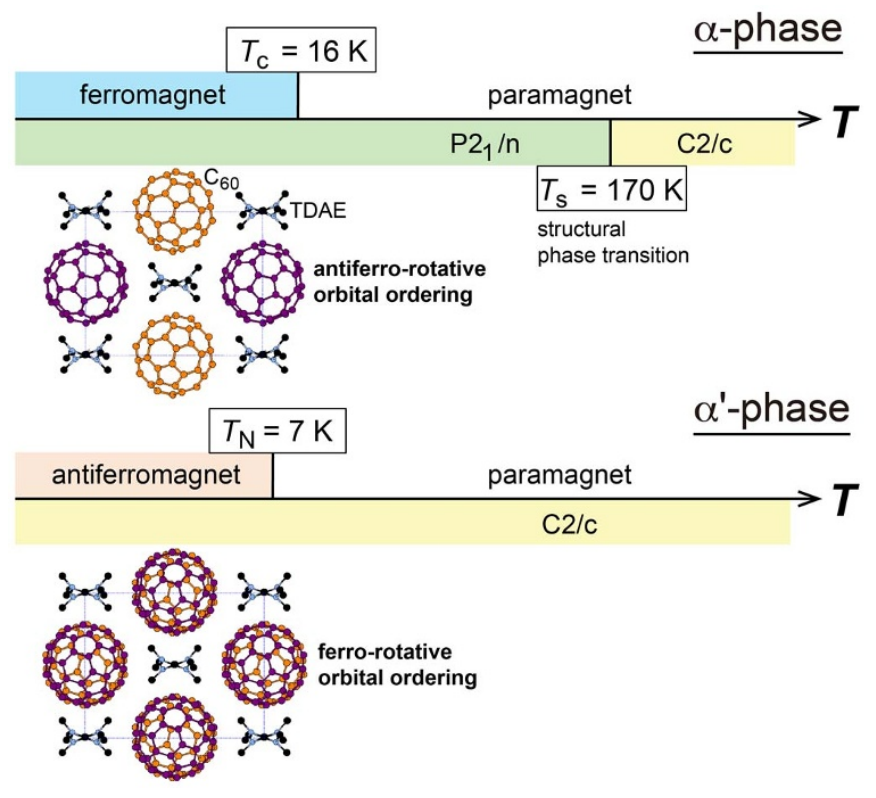

Figure $1 \mid$ Schematic phase diagram of $\alpha$ - and $\boldsymbol{\alpha}^{\prime}$-phase TDAE- $\mathrm{C}_{60}$. The low-temperature structure of the $\alpha$-phase has an antiferro-rotative arrangement for $\mathrm{C}_{60}$ within the $a b$-plane, while that of the $\alpha^{\prime}$-phase has a ferro-rotative arrangement.

produced by the pairing of a charged fullerene cage with a TDAE molecule. Thus, this system may be a good candidate for studying the correlation between the dielectric and magnetic properties in fullerene-based magnets.

\section{Results}

Figures $2 \mathrm{a}-2 \mathrm{~d}$ show the capacitance and dielectric loss of $\alpha$-phase TDAE- $\mathrm{C}_{60}$ as a function of temperature and frequency. At room temperature, the real part of the dielectric permittivity for the DC limit, $\varepsilon$, is found to be $\sim 79$. Both the capacitance and the dielectric loss clearly depended on the temperature and frequency. The $\varepsilon$ ' spectrum at fixed frequency exhibits a shoulder structure at a given temperature, and the dielectric loss reaches a maximum at this point (e.g. $\sim 180 \mathrm{~K}$ at $10 \mathrm{kHz}$ ). These fundamental characteristics may be considered as indicative of the Debye-type relaxation process for a permanent dipole moment. In addition, the shoulder structure of $\varepsilon$ ' and the peak in the dielectric loss shift to lower temperatures with decreasing frequency, as can be seen in Figs. $2 \mathrm{a}$ and $2 \mathrm{~b}$. Figures $2 \mathrm{c}$ and $2 \mathrm{~d}$ show the isothermal capacitance and dielectric responses, respectively, while typical Cole-Cole plots at fixed temperatures are shown in Fig. 2e. These plots can also be explained by the Debye-type relaxation of the dipole moments.

In this case, the Cole-Cole plots can be empirically analyzed by the well-known formula ${ }^{13}$ :

$$
\varepsilon^{*}(\omega)=\varepsilon^{\prime}(\omega)-j \varepsilon^{\prime \prime}(\omega)=\varepsilon(\infty)+\frac{\varepsilon(0)-\varepsilon(\infty)}{1+(j \omega \tau)^{1-\beta}},
$$

where $\varepsilon(0)$ and $\varepsilon(\infty)$ are the complex dielectric permittivity for the low- and high-frequency limits, respectively, $\tau$ is the relaxation time of the dipole moment, and $\beta$ is an empirically introduced parameter for the central angle of the circular arc of the Cole-Cole plot, which is defined as the multiplicity of the dispersion of relaxation ${ }^{13}$. By fitting the experimental data to this formula, the $\tau$ and $\beta$ values at each temperature can be determined. Figures $2 \mathrm{f}$ and $2 \mathrm{~g}$ show the Arrhenius plots for $\tau$ and the temperature dependence of $\beta$, respectively, for $\alpha$-TDAE- $C_{60}$. The temperature dependence of $\tau$ adheres well to the activation process from $300 \mathrm{~K}$ to $150 \mathrm{~K}$. It should be noted that $150 \mathrm{~K}$ nearly corresponds to the structural phase transition temperature $(T s \sim 170 \mathrm{~K})$ of $\alpha$-phase TDAE- $\mathrm{C}_{60}$. The solid line in Fig. $2 \mathrm{f}$ shows the fitted line to the activation formula: $\tau=\tau_{0} \exp \left(\frac{E_{a}}{k_{B} T}\right)$. The relaxation time, $\tau_{0}$, and the activation energy, $E_{\mathrm{a}}$, were found to be $4.0 \times 10^{-12} \mathrm{~s}$ and $273 \mathrm{meV}$, respectively. This activation energy is in agreement with the value reported by Pevzner et al. for $\mathrm{a} \mathrm{C}_{60}$ film ${ }^{14}$ and corresponds well to the results obtained for the energy difference between the hexagon-hexagon and hexagonpentagon contacts of neighboring $\mathrm{C}_{60}$ molecules $^{15}$. Importantly, while $\tau$ is apparently unchanged near the Ts, $\beta$ shows an anomaly in this temperature region. The very small $\beta$ value suggests a homogeneous relaxation process for an individual permanent electric dipole. In addition, the $\beta$ value decreases monotonically with decreasing temperature and exhibits temperature-independent behavior below the $T \mathrm{~s}$. The freezing of $\mathrm{C}_{60}$ 's rotation in $\alpha$-TDAE$\mathrm{C}_{60}$ is also evidenced below $150 \mathrm{~K}$ by NMR experiments ${ }^{16}$. Accordingly, the observed dielectric behavior should be responsible for the permanent dipole moments around the fullerene cages.

Next, from the Cole-Cole plot of $\alpha$-TDAE- $\mathrm{C}_{60}$ at $240 \mathrm{~K}$, the permittivity for the low-frequency and high-frequency limits, $\varepsilon(0)$ and $\varepsilon(\infty)$, were determined to be 79 and 3.1, respectively. To calculate the permanent dipole moment, the Clausius-Mossotti equation was then used. In this model, the contribution of molecular polarizability and permanent dipole moment in a crystal are included ${ }^{17}$ :

$$
\frac{\varepsilon-1}{\varepsilon+2}=\frac{N}{3 s_{0}}\left(4 \pi \varepsilon_{0} \alpha_{m}+\frac{\mu^{2}}{3 k_{B} T}\right),
$$

where $\varepsilon$ is the relative permittivity of the sample, $N$ is the molecular density of the sample, $\mu$ is the permanent dipole moment, $\alpha_{\mathrm{m}}$ is the molecular polarizability, $\varepsilon_{0}$ is the permittivity of vacuum, $k_{\mathrm{B}}$ is the Boltzmann constant, and $T$ is the temperature.

Owing to the presence of an eliminable permanent electric dipole at the high-frequency limit in $\alpha$-TDAE- $C_{60}$, the $\alpha_{\mathrm{m}}$ value was evaluated. Using the $\varepsilon(\infty)=3.1$ and $N=9.627 \times 10^{20} \mathrm{~cm}^{-3}$ values, the $\alpha_{\mathrm{m}}$ value was calculated to be $101 \AA^{3}$. The $\alpha_{m}$ of $C_{60}$ has been experimentally estimated by several authors and reported to be $76.5 \AA^{3}$ for isolated $\mathrm{C}_{60}{ }^{18}$ and $90 \AA^{3}$ for a $\mathrm{C}_{60}$ film ${ }^{14}$. Theoretical calculations, meanwhile, imply that $\alpha_{\mathrm{m}}=80-90 \AA^{3}$ for $\mathrm{C}_{60}{ }^{19}$. The larger $\alpha_{\mathrm{m}}$ value obtained for $\alpha$-TDAE- $C_{60}$ in this study suggests that it may include the contribution of the polarizability of the TDAE molecule. Using the experimentally obtained permittivity at the low-frequency limit, $\varepsilon(0)=79$, the $\mu$ value at $240 \mathrm{~K}$ was then determined by solving Eq. (2) and found to be $\mu=3.7 \mathrm{D}$. For the $\mathrm{C}_{60}$ film, an electric dipole moment as large as $0.9 \mathrm{D}$ was observed. It was suggested to originate from the pairing of interstitial oxygen and $\mathrm{C}_{60}$ molecules ${ }^{14}$. For alkalimetal-doped $\mathrm{C}_{60}$, permanent electric dipole moments (12-22 D) originating from the pairing of the mono cations of alkali metals and negatively charged $\mathrm{C}_{60}$ molecules ${ }^{20}$ have also been reported. In alkali-metal doped $\mathrm{C}_{60}, \mu$ depended on the doped metal. In $\alpha$-TDAE$\mathrm{C}_{60}$, one-electron charge transfer from TDAE to $\mathrm{C}_{60}$, which leads to the formation of the mono cation of TDAE and the mono anion of $\mathrm{C}_{60}$, was confirmed via magnetization ${ }^{10}, \mathrm{NMR}^{16}$, and photoemission spectroscopy (PES) experiments ${ }^{21}$. Thus, the observed small $\mu$ for the $\alpha$-phase may be due to the cancelation of each of the electric dipole moments.

Notably, the dielectric responses of the $\alpha^{\prime}$-phase crystals are completely different from those of the $\alpha$-phase. Figures $3 a$ and $3 b$ show the capacitance and dielectric loss of the $\alpha^{\prime}$-phase crystals as a function of temperature, respectively, while Figs. $3 c$ and $3 d$ show the isothermal dielectric responses as a function of frequency, respectively. The temperature dependence of the $\varepsilon^{\prime}$ value for the $\alpha^{\prime}$-phase is relatively weaker than that of the $\alpha$-phase. The Cole-Cole plots, meanwhile, show a more pronounced behavior, as can be seen in Fig. 3e. These results are the first evidence of different physical properties for two polymorphs near room temperature. The dielectric permittivity for the $\alpha^{\prime}$-phase at the low-frequency limit is as large 


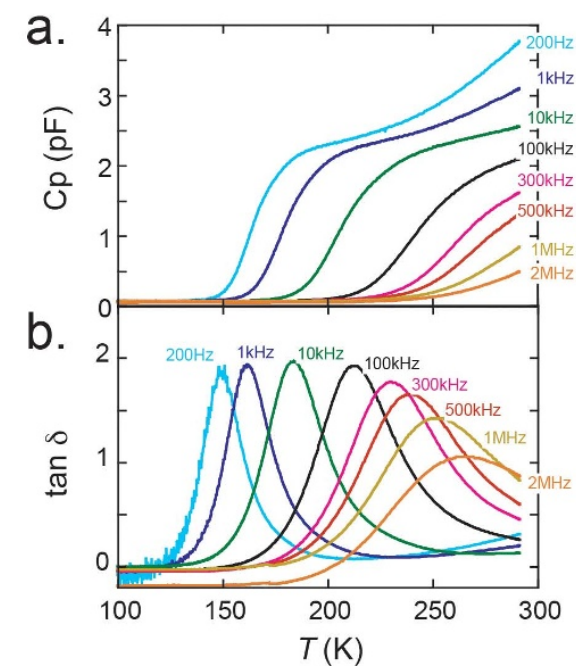

f.

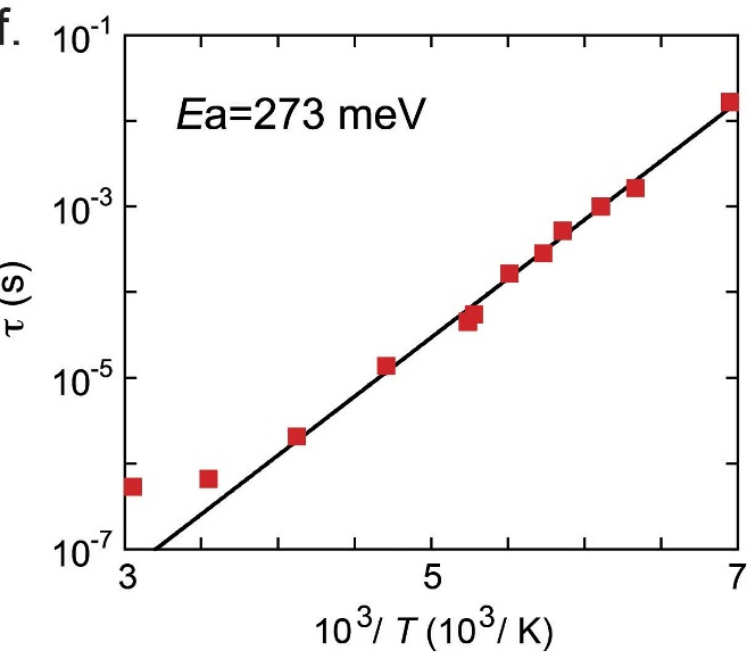

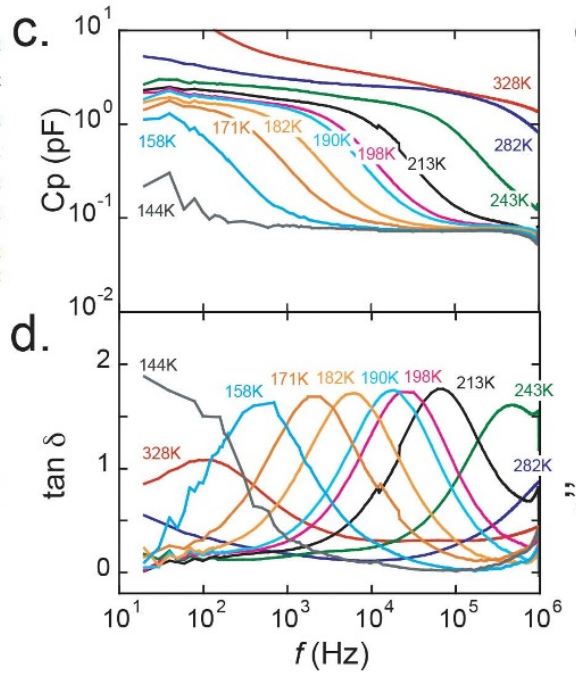

e.

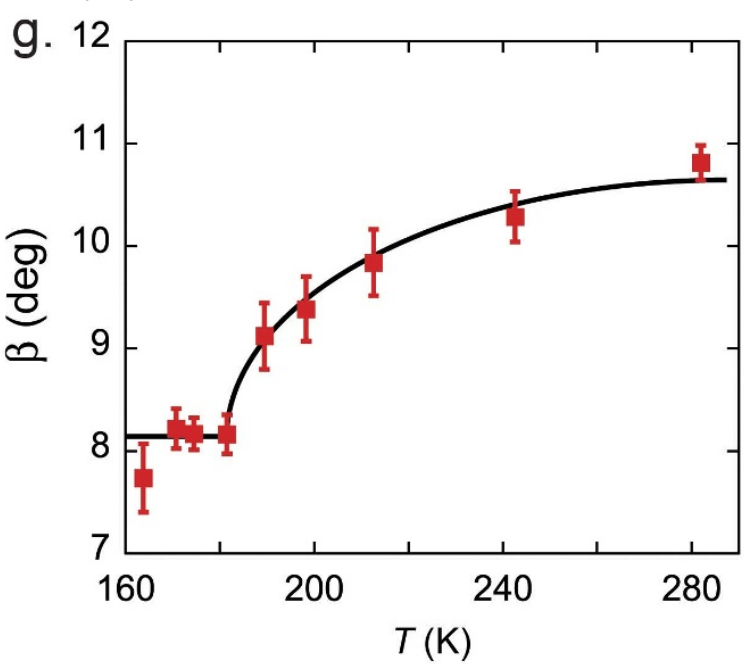

Figure $2 \mid$ Dielectric response of $\boldsymbol{\alpha}$-TDAE- $\mathrm{C}_{60}$. Temperature dependence of the capacitance (a) and dielectric loss (b) at various frequencies. Frequency dependence of the capacitance (c) and dielectric loss (d) at various temperatures. Cole-Cole plots at various temperatures (e). Arrhenius plots for the relaxation time $\tau$ (f) and the temperature dependence of $\beta(\mathrm{g})$ for $\alpha$-TDAE- $\mathrm{C}_{60}$. In (f), the solid line shows the fitted line to the activation formula $\tau=\tau_{0} \exp \left(\frac{E_{a}}{k_{B} T}\right)$ with $E \mathrm{a}=273 \mathrm{meV}$ and $\tau_{0}=4 \times 10^{-12} \mathrm{~s}$. In $(\mathrm{g})$, the solid line is a guide for the eye.
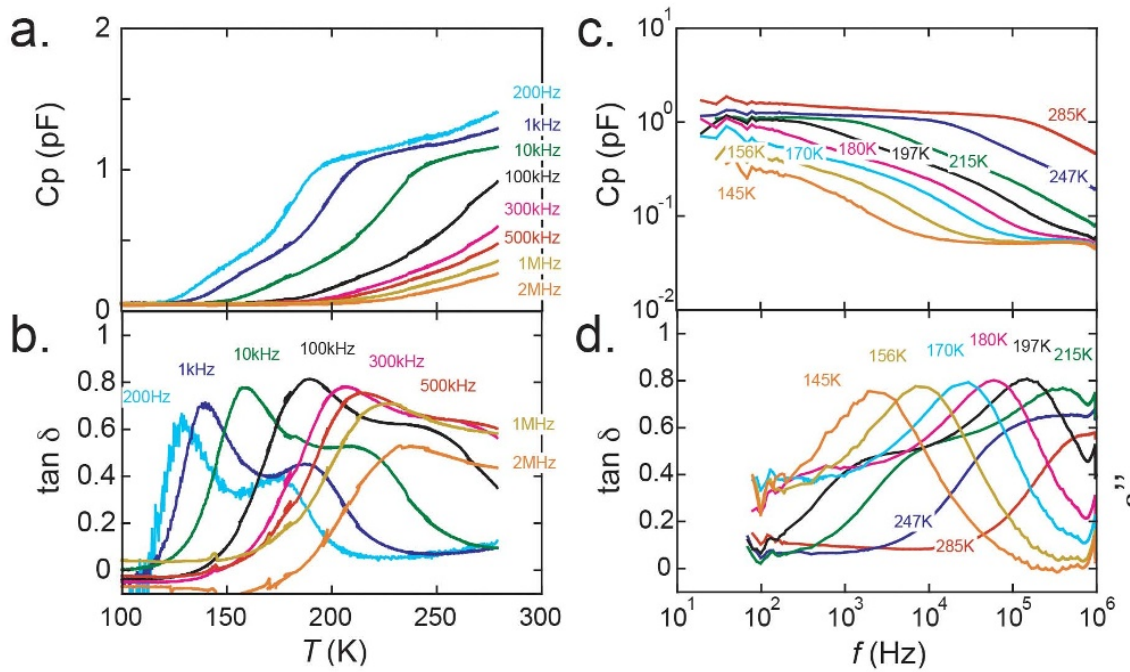

e.

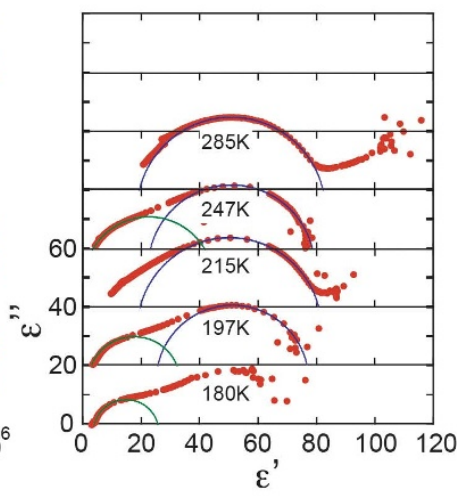

Figure $3 \mid$ Dielectric response of $\boldsymbol{\alpha}^{\prime}$-TDAE-C 6 . Temperature dependence of the capacitance (a) and dielectric loss (b) at various frequencies. Frequency dependence of the capacitance (c) and dielectric loss (d) at various temperatures. Cole-Cole plots at various temperatures (e). 
as that for the $\alpha$-phase, which can be reasonably understood by considering the same origin of the electric dipole. However, as can be seen from Fig. 3, it was not possible to reproduce the data using a dielectric function with a single component. The dielectric response of the $\alpha^{\prime}$-phase shows a broad distribution of relaxation times and the existence of multiple relaxation processes. For example, the ColeCole plots in Fig. 3e suggest the overlap of at least two dielectric functions. Moreover, the dielectric responses of the $\alpha^{\prime}$-phase seem to depend on the sample batch, while those of the $\alpha$-phase crystals did not (see Supplementary Fig. S1). This behavior strongly suggests a more complex environment for the local electric dipoles in the $\alpha^{\prime}$ phase crystal. Thus, the structural study indicates that the annealing procedure causes the differences in the LT structures for the two polymorphs (see Fig. 1). That is, a structural phase transition was not observed in the $\alpha^{\prime}$-phase. The different dielectric behavior for the two polymorphs implies that the annealing procedure for the asgrown sample primarily stabilizes the local environment around the molecules, thus resulting in homogeneous molecule displacement and orientational ordering, which lead to the structural phase transition (see below for further discussion).

The observed strong frequency dependence of dielectric properties may be supposed by a formation of dipolar glass phase at low temperatures $^{22}$. In the $\alpha$-TDAE- $\mathrm{C}_{60}$, however, the Cole-Cole plot is analyzed as one semicircle, which indicates that the relaxation can be described by single process. Even in the $\alpha$ '-phase, the relaxation may be described by a few processes. Thus, we may simply consider as a fluctuation of uniform dipole moment, not a formation of dipolar glass state, which should has a large distribution of relaxation time ${ }^{22}$. Contrary to the frequency dependence of dielectric response, the AC magnetic susceptibility for the $\alpha$-phase crystal shows no frequency dependence around $T_{\mathrm{c}}$, indicating no glass like transition. Moreover, the long-range magnetic ordered states in both crystal phases are essentially established by the magnetic resonance experiments, that is, the ferromagnetic resonance (FMR) in the $\alpha$-phase and the antiferromagnetic resonance (AFMR) in the $\alpha^{\prime}$-phase. Thus, we may not consider the spin-glass state for the magnetic ground state of TDAE$\mathrm{C}_{60}$.

\section{Discussion}

Specifically, in the RT structure (C2/c) of $\alpha$-TDAE-C 60 (Fig. 4), the TDAE molecules are located on the $4 \mathrm{e}$ site with a shift $(\sim 0.07 \AA)$ along the $b$-axis; here the center of the skeletal structure N2-C-C-N2 was used to represent TDAE in order to calculate the molecular shift. The shifting of the TDAE molecules is alternatively arranged along the $c$-axis, as depicted by the green arrows in Fig. $4 \mathrm{~b}$. In the LT structure, very weak changes in the absolute values of the shifts along the $b$-axis are observed (not shown). Moreover, the LT structure of the $\alpha$-phase permits the alternative shifting of the TDAE molecules along the $c$-axis in addition to the shifting along the $b$-axis, which is depicted by the red arrows in Fig. $4 \mathrm{~b}$. The absolute value of the shift along the $c$-axis is approximately $0.07 \AA$. In general, owing to the presence of the inversion symmetry on the $\mathrm{C}_{60}$ sites, the permanent electric dipole that was localized on $\mathrm{C}_{60}$ or centered at it was rejected. On the other hand, all of the TDAE molecules are cubically surrounded by eight $\mathrm{C}_{60}$ molecules, as shown in Fig. $4 \mathrm{a}$. Here it is assumed that both types of molecules have point charges, and the electric dipole moments originating from the pairing of a TDAE molecule and the surrounding $\mathrm{C}_{60}$ molecules are summed. As a result, a value of $1.48 \mathrm{D}$ for the electric dipole moment is obtained if only the shifting of the TDAE molecule along the $b$-axis is adopted, while a value of $2.95 \mathrm{D}$ is calculated if the contributions of the shifting of the TDAE molecule along both the $b$ - and $c$-axes are adopted. The merged electric dipole moment points along the $b$-axis for the former case and nearly along the $b+c$ direction for the latter case. While this model may be an oversimplification for estimating the exact $\mu$ value, the calculated $\mu$ value surprisingly reproduces the experimentally

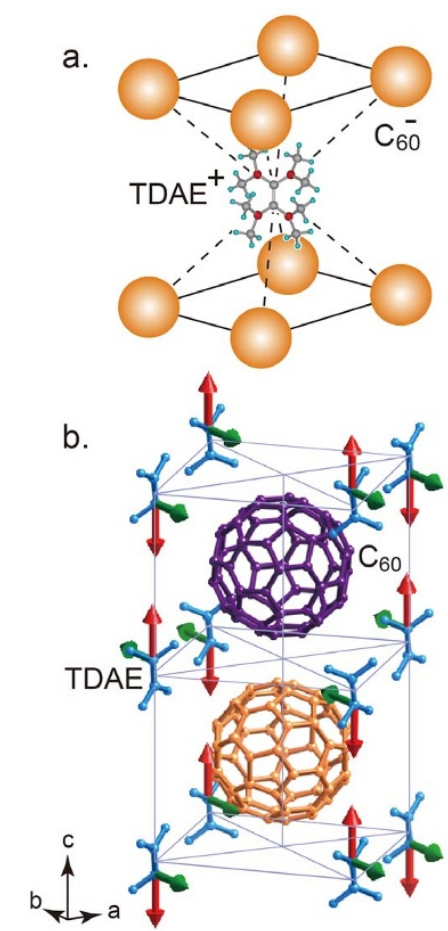

Figure 4 | Electric dipole moment and Low-temperature structure. Schematic view near the TDAE molecule, (a) which is surrounded cubically by eight $\mathrm{C}_{60}$ molecules. (b) Low-temperature structure and molecular shifting of the TDAE in $\alpha$-TDAE- $\mathrm{C}_{60}$.

obtained value. The present dielectric measurements validate the ratchet motion of $\mathrm{C}_{60}$ between two stable configurations with an energy separation of $E_{\mathrm{a}}$ at high temperature. Moreover, the LT structural analysis indicates that the $\mathrm{C}_{60}$ 's orientational ordering in the $\alpha$ phase works in close cooperation with the cubical distortion of the surrounding TDAE molecules ${ }^{11}$. Accordingly, it may be argued that, in the high-temperature region above the $T$ s, the ratchet motion of $\mathrm{C}_{60}$ occurs cooperatively with the dynamical movement of the TDAE molecule along the $c$-axis, which leads to the thermally fluctuating electric dipole moment. The precise evaluation of the electric dipole moment using the MOs of the cages with the JT distortion must be accomplished in the future.

For now, the information obtained in this study can be used to explain the effect of the annealing process on the two polymorphs of TDAE- $\mathrm{C}_{60}$. We propose that the JT distortion of the $\mathrm{C}_{60}$ molecule plays an important role in forming the crystals of TDAE- $\mathrm{C}_{60}$. Because the mono anion state of the $\mathrm{C}_{60}$ molecule has a prolate spheroidal shape, it has two types of rotational moments of inertia. Any $\mathrm{C}_{60}$ molecule rotating with respect to their long axis may have a higher probability of associating with TDAE molecules. In addition, during crystal formation, the molecular packing should be essential and prioritized between $\mathrm{C}_{60}$ and TDAE, and thus the $\mathrm{C}_{60}$ molecules should be arranged ferro-rotatively. However, to stabilize the exchange energy between the molecules after crystal formation and charge transfer, the $\mathrm{C}_{60}$ molecules will attempt to change their arrangements. During the annealing process, the antiferro-rotative arrangement should be stabilized because of the enlarged mutual distances between the molecules. Thus, there are two possible configurations for a stable magnetic arrangement ${ }^{5}$. The hightemperature ratchet motion of the $\mathrm{C}_{60}$ molecules between the two configurations is considered to be the origin of dielectric relaxation. Although the change in the molecular arrangement is not perceivable via X-ray structural analysis, the average distance of the unit cell is increased after the annealing procedure ${ }^{10,11}$. In the $\alpha$-phase, the structural phase transition simultaneously occurs with the rotational 
ordering of the $\mathrm{C}_{60}$ molecules, and ferromagnetism appears because of symmetry breaking. On the other hand, the $\alpha^{\prime}$-phase maintains the high-temperature structure after the suspension of $\mathrm{C}_{60}$ rotation as indicated by its anti-ferromagnetism.

\section{Conclusion}

In conclusion, dielectric measurements were obtained for the two polymorphs of TDAE- $\mathrm{C}_{60}$, and differences in their dielectric responses in the high-temperature region were observed. This dielectric study on fullerene-based magnets reveals that the low-temperature magnetic characteristics of the two polymorphic crystals were determined by the properties of the crystals at room temperature and the close correlation between their dielectric and magnetic properties. The magnetism of fullerene magnets is responsible for the spin localized on the $\mathrm{C}_{60}$. The control of the orientation of $\mathrm{C}_{60}$ molecules should allow modifying the electronic overlap between the neighboring molecular orbitals, which leads to the possible magnetic interaction (ferromagnetic or antiferromagnetic) between the spins. The present study shows the potential for manipulating the molecular orientation, that is, the magnetism, of $\mathrm{C}_{60}$ molecule by the electric field. We anticipate that fullerene magnets will be leading candidates for future molecular devices.

\section{Methods}

The single crystals were prepared by the usual diffusion method ${ }^{9-11}$, and several crystals obtained from different sample batches were carefully selected. Prior to the magnetic and the dielectric experiments, the crystals were examined by X-ray diffraction, and those with no twinning or contamination by other phases were chosen for further evaluation. X-ray diffraction measurements were performed using a Rigaku RAXIS-IV imaging plate diffractometer with graphite monochromated Mo$\mathrm{K} \alpha$ radiation. The magnetizations were measured using a Quantum Design superconducting interference device (SQUID), and a four-probe method was used to obtain the dielectric responses. The plate-shaped crystals were placed in a cryostat and cooled with liquid ${ }^{4} \mathrm{He}$ and ${ }^{3} \mathrm{He}$, thus enabling the measurement of the dielectric response from $400 \mathrm{~K}$ to $0.5 \mathrm{~K}^{12}$. The sample temperature was monitored using a Cernox thermometer. The typical size of the crystals was $1.5 \times 1.5 \times 0.5 \mathrm{~mm}$, and the thickness direction corresponded to the crystal $a$-axis. The electric field was applied nearly parallel to the $a$-axis. The capacitance and the dielectric loss were directly obtained using an LCR impedance meter (Agilent E4980A).

1. Kahn, O. Molecular Magnetism [Kahn, O. (ed)] (Wiley-VCH, New York, 1993).

2. Kawamoto, T. A theoretical model for ferromagnetism of TDAE- $\mathrm{C}_{60}$. Solid State Commun. 101, 231-235 (1997).

3. Andreoni, W. The Physics of Fullerene-Based and Fullerene-Related Materials [Andreoni, W. (ed)] (Kluwer Academic Publishers, 2000).

4. Allemand, P. M. et al. Organic molecular soft ferromagnetism in a fullerene $\mathrm{C}_{60}$. Science 253, 301-302 (1991).

5. Narymbetov, B. et al. Origin of ferromagnetic exchange interactions in a fullereneorganic compound. Nature 407, 883-885 (2000).

6. Ishii, $\mathrm{K}$. et al. Structural phase transition in the ammoniated alkali $\mathrm{C}_{60}$ compound $\left(\mathrm{NH}_{3}\right) \mathrm{K}_{3} \mathrm{C}_{60}$. Phys. Rev. B 59, 3956-3960 (1999).

7. Margadonna, S. et al. Orientational ordering of $\mathrm{C}_{60}$ in the antiferromagnetic $\left(\mathrm{NH}_{3}\right) \mathrm{K}_{3} \mathrm{C}_{60}$ phase. Phys. Rev. B 64, 132414(4 pages) (2001).

8. Jaglicic, Z., Trontelj, Z., Pirnat, J. \& Blinc, R. Magnetic properties of TDAE-C 60 single crystal and powder samples: The influence of thermal annealing. Solid State Commun. 101, 591-595 (1997).

9. Kambe, T., Kajiyoshi, K., Fujiwara, M. \& Oshima, K. Antiferromagnetic ordering driven by the molecular orbital order of $\mathrm{C}_{60}$ in $\alpha^{\prime}$-Tetra-Kis-(Dimethylamino)Ethylene-C 60 . Phys. Rev. Lett. 99, 177205(4 pages) (2007).
10. Kambe, T., Nogami, Y. \& Oshima, K. Annealing effects on the magnetic and structural properties of single-crystal TDAE-C 60 . Phys. Rev. B 61, R862-R865 (2000).

11. Fujiwara, M., Kambe, T. \& Oshima, K. Structural differences in two polymorphs of tetra-kis-(dimethylamino)-ethylene- $\mathrm{C}_{60}$ : An x-ray diffraction study. Phys. Rev. B 71, 174424(7 pages) (2005).

12. Kajiyoshi, K., Kambe, T., Tamura, M. \& Oshima, K. Antiferromagnetic ground state in organic quasi-1D ferromagnet $\gamma$-phase para-nitrophenyl nitronyl nitroxide. J. Phys. Soc. Jpn. 75, 074702(7 pages) (2006).

13. Cole, K. S. \& Cole, R. H. Dispersion and absorption in dielectrics I. Alternating current characteristics. J. Chem. Phys. 9, 341 (1941).

14. Pevzner, B., Hebard, A. F. \& Dresselhaus, M. S. Role of molecular oxygen and other impurities in the electrical transport and dielectric properties of $\mathrm{C}_{60}$ films. Phys. Rev. B 55, 16439-16449 (1997).

15. Prassides, K. Neutron scattering and $\mu$ SR studies of fullerene and their derivatives. Phys. Scr. T49, 735-741 (1993).

16. Arcon, D., Dolinsek, J. \& Blinc, R. ${ }^{13} \mathrm{C}$ NMR of the organic ferromagnet TDAEC60. Phys. Rev. B 53, 9137-9142 (1996).

17. Frohlich, H. Theory of Dielectrics: Dielectric constant and Dielectric loss [Frohlich, H. (ed)] (Oxford: Clarendon Press, 1986).

18. Antoine, R. et al. Direct measurement of the electric polarizability of isolated $\mathrm{C}_{60}$ molecules. J. Chem. Phys. 110, 9771 (1999).

19. Gamba, Z. Polarizability and intermolecular potential of $\mathrm{C}_{60}$. Phys. Rev. B 57, 1402 (1998).

20. Antoine, R. et al. Electric dipole moment and charge transfer in alkali- $\mathrm{C}_{60}$ molecules. Eur. Phys. J. D 12, 147-151 (2000)

21. Yamaoka, H. et al. Electronic state of an organic molecular magnet: Soft $\mathrm{x}$-ray spectroscopy study of $\alpha-$ TDAE $\mathrm{C}_{60}$ single crystal. Phys. Rev. B 84, 161404(R)(4 pages) (2011).

22. Choudhury, D. S. et al. Tuning of dielectric properties and magnetism of $\mathrm{SrTiO}_{3}$ by site-specific doping of Mn. Phys. Rev. B 84, 125124(7 pages) (2011).

\section{Acknowledgments}

We thank M. Fujiwara, K. Kajiyoshi, K. Ishida, and the members of the Quantum Material Laboratory for supporting these experiments. We are also grateful to Y. Nogami for stimulating discussions on the structure and to $\mathrm{N}$. Ikeda for stimulating discussions on the dielectric properties. This research is partly supported by a Grant-in-Aid for Scientific Research from the Japanese Ministry of Education, Culture, Sports, Science and Technology, Japan (23340104).

\section{Author contributions}

T. K. and K. O. planned the study. T. K. synthesized the samples and collected experimental data. All authors discussed the results and wrote the manuscript.

\section{Additional information}

Supplementary information accompanies this paper at http://www.nature.com/ scientificreports

Competing financial interests: The authors declare no competing financial interests.

How to cite this article: Kambe, T. \& Oshima, K. Dynamically fluctuating electric dipole moments in fullerene-based magnets. Sci. Rep. 4, 6419; DOI:10.1038/srep06419 (2014)

This work is licensed under a Creative Commons Attribution-NonCommercialNoDerivs 4.0 International License. The images or other third party material in this article are included in the article's Creative Commons license, unless indicated otherwise in the credit line; if the material is not included under the Creative Commons license, users will need to obtain permission from the license holder in order to reproduce the material. To view a copy of this license, visit http:// creativecommons.org/licenses/by-nc-nd/4.0/ 